ఠ

\title{
miR-222-3p promotes osteosarcoma cell migration and invasion through targeting TIMP3
}

This article was published in the following Dove Press journal: OncoTargets and Therapy

\author{
Jianping Guo \\ Quanxiang Liu \\ Zengxin $\mathrm{Li}$ \\ Haifeng Guo \\ Changshuang Bai \\ Fajia Wang
}

Department of Orthopaedic Surgery, Affiliated Hospital of Beihua University, Jilin I320I I, PR China
Correspondence: Quanxiang Liu Department of Orthopaedic Surgery, Affiliated Hospital of Beihua University, No. 12 Jiefang Middle Road, Jilin I320I I, PR China

Email 249602238@qq.com
Background: Abnormal expression of miRNAs has been reported in osteosarcoma (OS), and miR-222-3p levels have been found to be increased in the serum of OS patients. However, the exact role of miR-222-3p in OS remains unclear. In the present study, we aimed to identify the molecular mechanism underlying the role of miR-222-3p in the development of OS.

Methods: We examined the expression level of miR-222-3p in OS tissues and OS cells using reverse-transcription quantitative PCR (RT-qPCR) analysis. MTT, colony formation, and transwell invasion assays were used to analyze the effects of miR-222-3p on the proliferation and invasion ability of OS cells. Luciferase reporter gene assays were used to confirm the target gene of miR-222-3p in OS cells. Tumor xenografts were then used to investigate the role of miR-222-3p in OS growth in vivo.

Results: The data of the present study demonstrated that miR-222-3p levels were increased in OS tissues and OS cells. Downregulation of miR-222-3p significantly inhibited the proliferation, migration, and invasion of OS cells in vitro. Further analysis revealed that tissue inhibitors of metalloproteinases 3 (TIMP3) is one of the functional target genes of miR-222-3p, and inhibition of TIMP3 efficiently rescues the blocking of cell proliferation and invasion mediated by miR-222-3p inhibitor in OS cells.

Conclusion: Our findings constitute evidence that miR-222-3p promotes OS cell proliferation and invasion through targeting TIMP3 mRNA and provide novel insight into the mechanism underlying the development of OS.

Keywords: microRNA-222-3p, osteosarcoma, tissue inhibitor of metalloproteinases 3, migration, invasion

\section{Introduction}

Osteosarcoma (OS) is the most frequent malignant primary bone tumor in adolescents and is characterized by high rates of metastasis and recurrence. ${ }^{1,2}$ Recently, advances in the diagnosis and treatment of OS have improved patient outcomes; however, due to its high invasiveness and propensity for metastasis, the prognosis of OS remains poor. ${ }^{3,4}$ Previous studies revealed that gene therapy may be a rational and promising strategy for patients with various cancers, including OS. ${ }^{5-8}$ However, the mechanisms underlying OS progression and metastasis remain obscure. Thus, elucidating these underlying molecular mechanisms and identifying novel diagnostic biomarkers is crucial for OS treatment.

miRNAs are a class of endogenous noncoding RNAs (18-25 nucleotides in length), which regulate gene expression via binding to the 3 '-untranslated region (UTR) of target mRNAs posttranscriptionally. ${ }^{9}$ It has been reported that miRNAs are ectopically expressed in various diseases, including OS. ${ }^{10-12}$ A recent study demonstrated that miR222 was abnormally expressed in OS patients. ${ }^{13}$ However, the role of miR-222 in OS 
remains unknown. Therefore, determining the exact role of miR-222 in OS progression may contribute to the development of an effective therapeutic strategy for OS.

Tissue inhibitors of metalloproteinases (TIMPs), a family of endogenous protease inhibitors, control extracellular matrix (ECM) proteolysis through inhibition of matrix metalloproteinases (MMPs). ${ }^{14}$ It has been reported that TIMPs also inhibit a disintegrin and metalloproteinases with thrombospondin motifs proteins. ${ }^{15}$ Previous studies demonstrated that TIMP3 deficiency enhanced cell proliferation, angiogenesis, migration, and invasion in various types of cancer, including lung, colorectal, and prostate cancers. ${ }^{16-18}$ Moreover, overexpression of TIMP3 inhibits invasion and promotes apoptotic cell death in melanoma cell lines, ${ }^{19}$ whereas low expression of TIMP3 is implicated in OS metastasis. ${ }^{14}$

In the present study, we investigated the role of miR$222-3 p$ in OS and its mechanism of action. The findings were confirmed using a luciferase assay. In addition, the function of miR-222-3p was examined in vitro and in vivo, and the association between miR-222-3p and TIMP3 was investigated in OS patient tissues. The aim of the study was to elucidate the mechanism underlying OS development and progression and provide novel insights into its treatment.

\section{Materials and methods}

\section{Tissue specimens}

We selected 30 paired OS and adjacent non-OS tissues from the Affiliated Hospital of Beihua University. The histopathological subtypes of the OS samples included 15 osteoblastic, nine chondroblastic, and six fibroblastic cases. All the tissues were stored in liquid nitrogen until use. None of the patients received any treatment, such as chemotherapy or radiotherapy, prior to surgery. The age of patients was 10-65 years, and they included 19 men and 11 women. All adult patients and a parent for patients under 18 years of age provided written informed consent, and this study was conducted in accordance with the latest revision of the principles outlined in the Declaration of Helsinki. The study protocol was approved by the Clinical Research Ethics Committee of Beihua University (Jilin, China).

\section{Cell culture and transfection}

OS cell lines (MG-63, HOS, U2OS, and Saos-2) and the normal osteoblast cell line hFOB were purchased from Tianjin Sai'er Biotechnology Co. Ltd. (Tianjin, China). All OS cells were cultured in RPMI 1640 medium (Thermo Fisher Scientific, Waltham, MA, USA) supplemented with 10\% FBS (Thermo Fisher Scientific), and hFOB cells were cultured in DMEM with 10\% FBS. Cultures were maintained in a humidified atmosphere at $37^{\circ} \mathrm{C}$ with $5 \% \mathrm{CO}_{2}$.

miR-222-3p inhibitor and control inhibitor were purchased from RiboBio (Guangzhou, China). TIMP3-specific small interfering RNAs (si-TIMP3) and control small interfering RNAs (si-control) were purchased from GeneChem (Shanghai, China). Cells were cultured to $\sim 80 \%$ confluence, followed by transfection with miR-222-3p inhibitor ( $50 \mathrm{nM}$ ) or control inhibitor using Lipofectamine 2000 reagent (Thermo Fisher Scientific) according to the manufacturer's protocol. The cells were cultured in transfection media for 6 hours, and then the media were replaced by complete medium for the following assays.

\section{Reverse-transcription quantitative PCR (RT-qPCR) analysis}

Total RNA was extracted from OS cells using TRIzol reagent (Thermo Fisher Scientific) according to the manufacturer's instructions. Then, complementary DNA was synthesized using the Reverse Transcriptase M-MLV kit with random primers (TaKaRa, Dalian, China). GAPDH or U6 was used as an internal control. The relative mRNA and miRNA expressions were quantified using the $2^{-\Delta \Delta \mathrm{Cq}}$ method. The primers used were as follows: GAPDH forward 5'-ATC ACC ATC TTC CAG GAG GGA-3' and reverse $5^{\prime}$-CCT TCT CCA TGG TGG TGA AGA C-3'; U6 forward 5'-CTC GCT TCG GCA GCA CA-3' and reverse 5'-AAC GCT TCA CGA ATT TGC GT-3'; TIMP3 forward 5'-ACC GAG GCT TCA CCA AGA TG- $3^{\prime}$ and reverse $5^{\prime}$-CAT CAT AGA CGC GAC CTG TCA-3'; miR-222-3p forward 5'-AGC TAC ATC TGG CTA CTG GGT-3' and reverse 5'-GCG AGC ACA GAA TTA ATA CGA C-3'.

\section{Cell viability assay}

OS cells $\left(1 \times 10^{3}\right.$ per well) were seeded in 96-well culture plates (Corning, Shanghai, China), and then treated with miR-222-3p inhibitor or control inhibitor. A total of $20 \mu \mathrm{L}$ of MTT reagent was added to each well and incubated for 4 hours. The supernatant was discarded, and $150 \mu \mathrm{L}$ of dimethyl sulfoxide was added to dissolve formazan crystals by swirling gently. The absorbance was measured at $492 \mathrm{~nm}$ using a microplate reader.

\section{Colony formation assay}

The transfected OS cells were harvested and seeded at a density of 250 cells per well in 12 -well plates and incubated at $37^{\circ} \mathrm{C}$ with $5 \% \mathrm{CO}_{2}$ in a humidified incubator for 2 weeks. During colony growth, the culture medium was replaced every 3 days. The colony number in each well was counted and calculated. 


\section{Wound healing assay}

The OS cells were treated as indicated, seeded in six-well plates $\left(10^{4} /\right.$ well $)$ and cultured to $100 \%$ confluence. Subsequently, a wound was created in the cell monolayer using a sterile plastic micropipette tip. The cells were then washed by PBS and cultured for another 24 hours in serum-free medium. Images were captured at different time points ( 0 and 24 hours) under a light microscope to assess the rate of gap closure.

\section{Transwell cell invasion assay}

For the invasion assay, we performed the experiment using BioCoat Matrigel chamber according to manufacturer's protocol. Cells $\left(5 \times 10^{4}\right)$ were seeded in the upper chamber with FBS-free medium. The bottom chamber contained medium supplemented with $10 \%$ FBS. After incubation for 24 hours, the cells on the upper surface of the membrane were removed with a cotton swab and invading cells were stained with crystal violet solution. The number of stained cells was counted in five random fields under a microscope.

\section{Western blotting}

The cells or tissues were lysed using RIPA buffer (Solarbio, Beijing, China). The lysis solution was collected after centrifugation at $12,000 \times \mathrm{g}$ for 5 minutes at $4^{\circ} \mathrm{C}$. The protein concentration was determined using the $\mathrm{BCA}$ assay. Total protein $(10 \mu \mathrm{g})$ was loaded in 15\% SDS-PAGE gels and transferred to polyvinylidene fluoride membranes. The membrane was blocked with $5 \%$ skimmed milk overnight, and then incubated with primary antibody for 1 hour at room temperature. After washing, the membranes were incubated with a secondary antibody for 1 hour at room temperature. The immunoblots were acquired using Immobilon Western Chemiluminescent HRP Substrate kit (Millipore, Beijing, China) according to the manufacturer's protocol. The antibodies using in the present study were as follows: rabbit anti-TIMP3 (1:2,000; ProteinTech Group, Inc., Chicago, IL, USA) and mouse anti$\beta$-actin (1:3,000; ProteinTech Group, Inc.). Goat anti-rabbit and anti-mouse IgGs (ProteinTech Group, Inc.) were used as secondary antibodies diluted at 1:5,000.

\section{Luciferase reporter assay}

The TIMP3 3'-UTR containing wild-type or mutant miR222-3p seed sequence fragment was cloned and constructed in the PGL3-control with $X b a \mathrm{I} / F s e \mathrm{I}$ (NEB, Ipswich, MA, USA). The primers used were as follows: TIMP3 3'-UTR wild-type forward 5'-CTA GTC TAG AAG TGG ACA TCA GTG TCT TC-3' and reverse 5'-GGG GGC CGG CCT GTC TCA GCT ACA TAA ATG-3'; TIMP3 3'-UTR mutant type $5^{\prime}$-CTA GTC TAG AAG TGG ACA TCA GTG
TCT TC-3' and reverse $5^{\prime}$-GGG GGC CGG CCT GTC TCA GCA GTA TAA ATG-3'. OS cells were seeded into 24-well plates and cotransfected with luciferase reporter plasmids using Lipofectamine 2000 (Thermo Fisher Scientific) when the cells were $70 \%$ confluent. After 36 hours, luciferase activities were detected using the Luciferase Assay System (Promega Corporation, Fitchburg, WI, USA) with a GloMax luminometer (Promega), and the results were normalized against pRL-TK activity.

\section{Xenograft tumor model}

$\mathrm{BALB} / \mathrm{c}$ nude male mice, 5-week-old and weighing 20-25 g, were used for the in vivo tumor assay. OS cells $\left(2 \times 10^{6}\right)$, stably transfected the miR-222-3p inhibitor or miR-control inhibitor $(\mathrm{NC})$, were suspended in $100 \mu \mathrm{L}$ of serum-free DMEM/ Matrigel (1:1). Next, the cells were injected subcutaneously into the flank of each mouse. Tumor volumes were measured weekly and calculated using the formula: Volume $\left(\mathrm{mm}^{3}\right)=$ $1 / 2$ (length $\times$ width). ${ }^{2}$ The mice were sacrificed by cervical dislocation under anesthesia with diethyl ether after 30 days. The tumor tissues were harvested, weighed, and used to determine miR-222-3p and TIMP3 expression. The animal experiments were approved by the Laboratory Animal Ethics Committee of Beihua University (Jilin, China) and followed the Regulations for the Administration of Affairs Concerning Experimental Animals approved by the State Council and promulgated by Decree No 2 of the State Science and Technology Commission (1988).

\section{Statistical analysis}

The results were analyzed using Student's $t$-test and ANOVA. The correlation between miR-222-3p and TIMP3 in clinical OS tissue was analyzed with Pearson's correlation coefficient. The statistical significance was assessed by comparing mean values $( \pm \mathrm{SD})$ and was assumed for $* P<0.05$ and $* * P<0.01$.

\section{Ethics approval}

This study was approved by the Clinical Research Ethics Committee of Beihua University and the Laboratory Animal Ethics Committee of Beihua University (Jilin, China).

\section{Results miR-222-3p is upregulated in OS}

To identify the potential role of miR-222-3p in OS, we detected the expression of miR-222-3p by RT-qPCR in 30 pairs of OS tissues. The expression levels of miR222-3p in OS tissues were significantly higher compared with those in the corresponding normal tissues (Figure 1A). 
Moreover, miR-222-3p expression levels in OS cell lines (HOS, MG-63, Saos-2, and U-2OS) were increased compared with the normal human osteoblast cell line hFOB (Figure 1B). Considering that U-2OS and MG-63 cells exhibited higher expression of miR-222-3p among the four OS cell lines, HOS and U-2OS cells were selected to perform the following experiments. Our data demonstrated that miR-222-3p expression is increased in both OS tissues and cell lines, suggesting that its upregulation may be associated with OS progression.

\section{Inhibition of miR-222 in OS cells decreases their proliferation and colony formation ability}

To explore the effect of miR-222-3p on OS cell proliferation, miR-222-3p inhibitor or NC was transfected into MG-63 and U-2OS cells. RT-qPCR revealed that miR-222-3p inhibitor decreased the miR-222-3p expression in U-2OS and MG-63 cells (Figure 2A and B). Our data revealed that downregulation of miR-222-3p suppressed the proliferation of MG-63 and U-2OS cells compared with NC (Figure 2C and D). In addition, the colony formation assay revealed that downregulation of miR-222-3p decreased the colony formation ability of OS cells (Figure 2E and F). Thus, it was concluded that miR-222-3p increases the proliferation and colony formation ability of OS cells.

\section{Inhibition of miR-222 suppresses migration and invasion ability of OS cells}

To investigate the effects of miR-222-3p on the migratory and invasive behavior of OS cells in vitro, we performed wound healing and invasion assays. Our results demonstrated that
OS cells treated with miR-222-3p inhibitor exhibited a significant reduction in migration capacity compared with cells transfected with miR-NC (Figure 3A and B). Furthermore, ectopic expression of miR-222-3p induced a marked decrease in the invasion ability of U-2OS and MG-63 cells compared with cells transfected with miR-NC (Figure $3 \mathrm{C}$ and D). Taken together, these results suggest that inhibition of miR-222 suppresses migration and invasion of OS cells.

\section{TIMP3 is a direct target of miR-222-3p}

We next examined the potential targets of miR-222-3p. TargetScan 7.0, miRanda, and Pictar were used to predict miR222-3p target genes. TIMP3 attracted our attention among the candidate target genes (Figure 4A), and we validated whether miR-222-3p could directly target the 3 '-UTR of TIMP3 using luciferase reporter assays. Our data revealed that miR-222-3p significantly inhibited the firefly luciferase activity of pGL3TIMP3-wt in OS cells. By contrast, there was no such effect when the seed sequence was mutated (Figure 4B and C). Furthermore, Western blotting was performed to determine the effect of miR-222-3p on TIMP3 expression at the protein level. Overexpression of miR-222-3p significantly reduced TIMP3 expression in OS cells. Conversely, the expression of TIMP3 was upregulated when using miR-222-3p inhibitor in OS cells (Figure 4D and E). These results suggest that TIMP3 is one of the direct target genes of miR-222-3p in OS.

\section{TIMP3 expression is inversely correlated with miR-222-3p expression in OS tissues} As TIMP3 was confirmed as a target gene of miR-222-3p in OS cells, we determined its expression in OS and corresponding
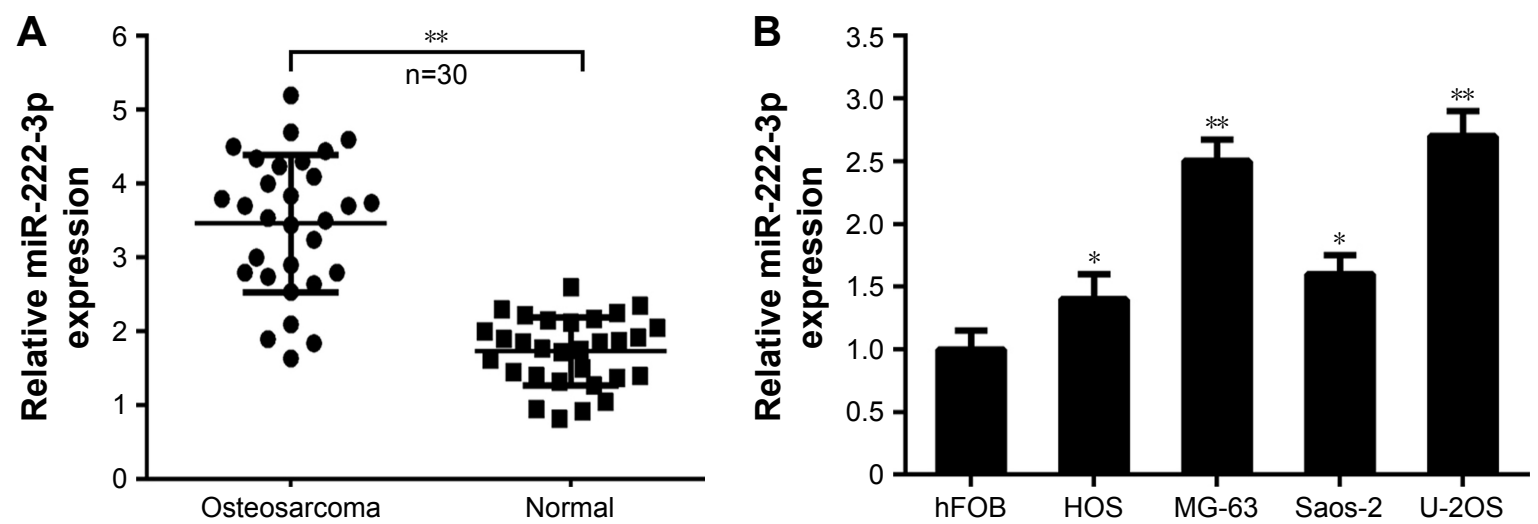

Figure I miR-222-3p is upregulated in OS tissues and cell lines.

Notes: (A) miR-222-3p mRNA levels were examined by qRT-PCR analysis in 30 cases of clinical OS tissues and paired peritumor tissues. **P $<0.01$. (B) Gene expression levels of miR-222-3p were compared between human osteosarcoma cell lines (HOS, MG-63, Saos-2, and U-2OS) and normal human osteoblast cell hFOB by qRT-PCR. $* P<0.05 ; * * P<0.01$.

Abbreviations: OS, osteosarcoma; RT-qPCR, reverse-transcription quantitative PCR. 

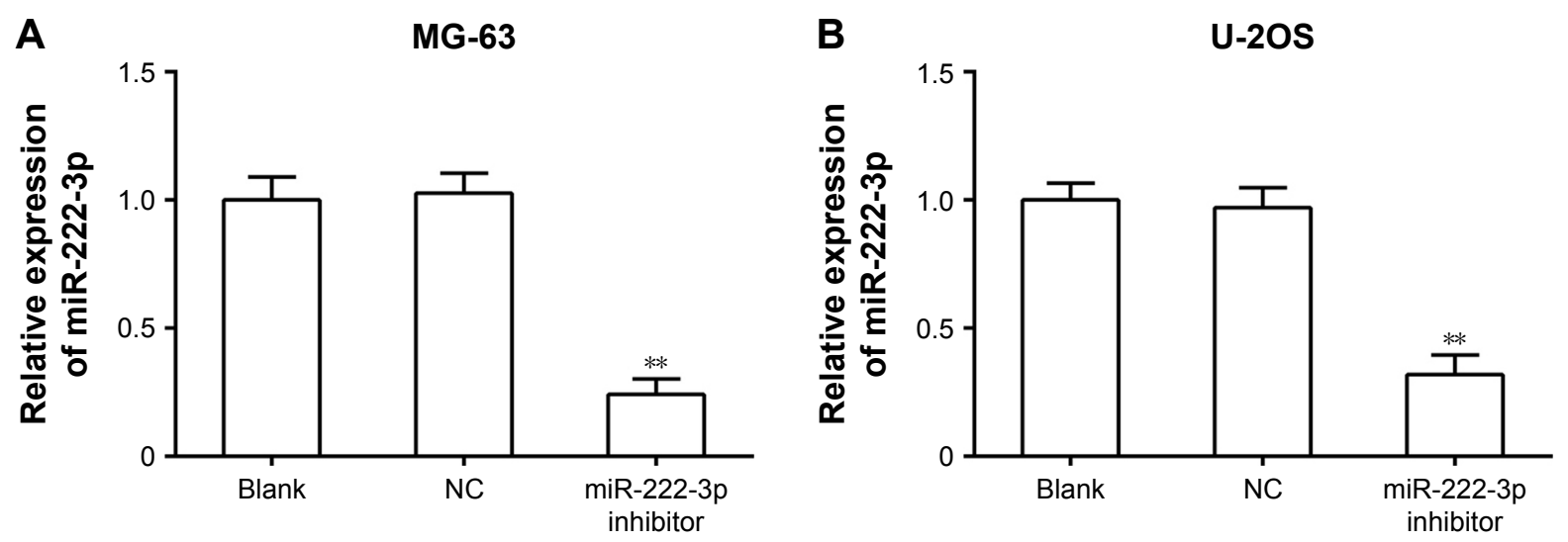

C

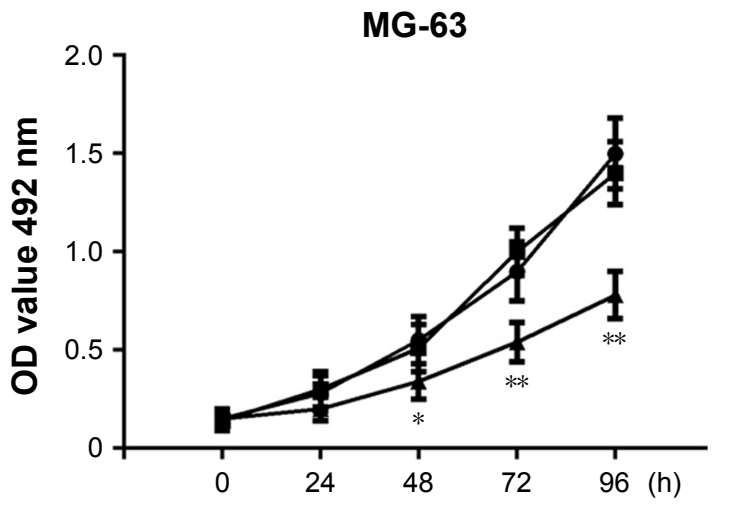

D

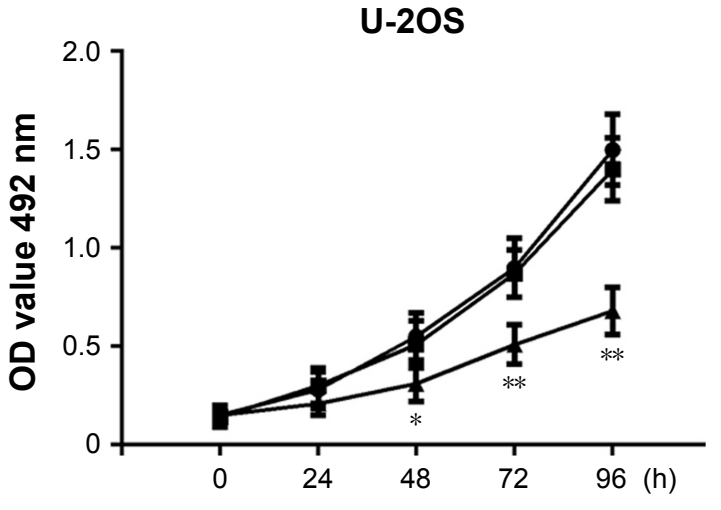

Blank

NC

$\leftarrow$ miR-222-3p inhibitor

$\mathbf{E}$

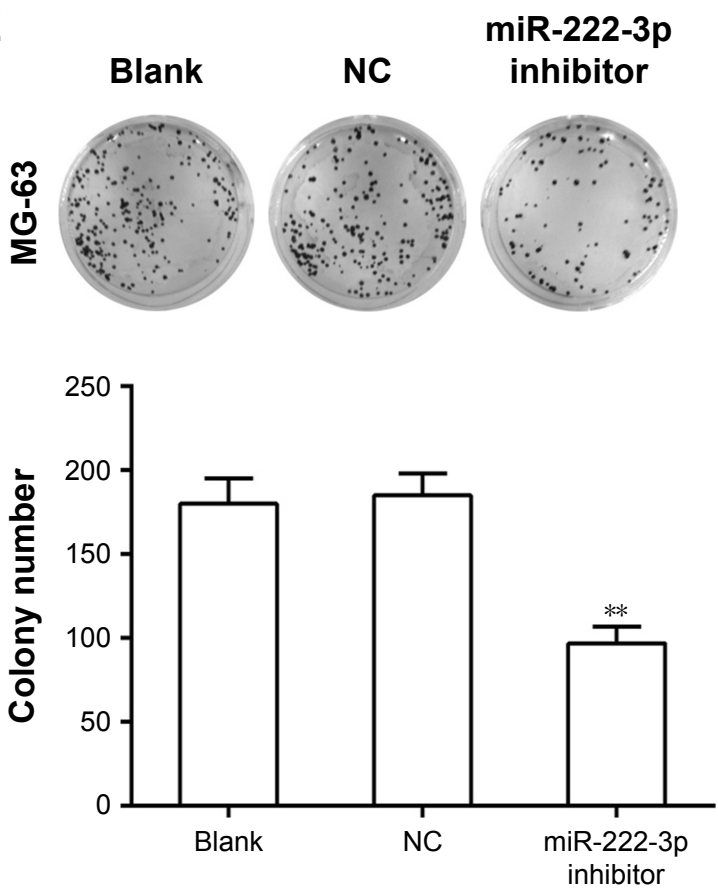

$\mathbf{F}$

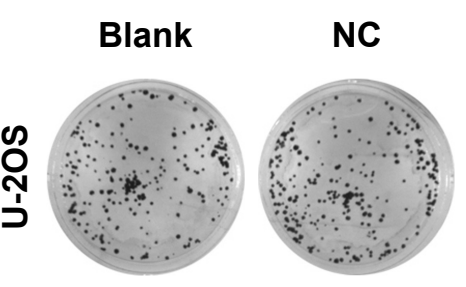

miR-222-3p inhibitor
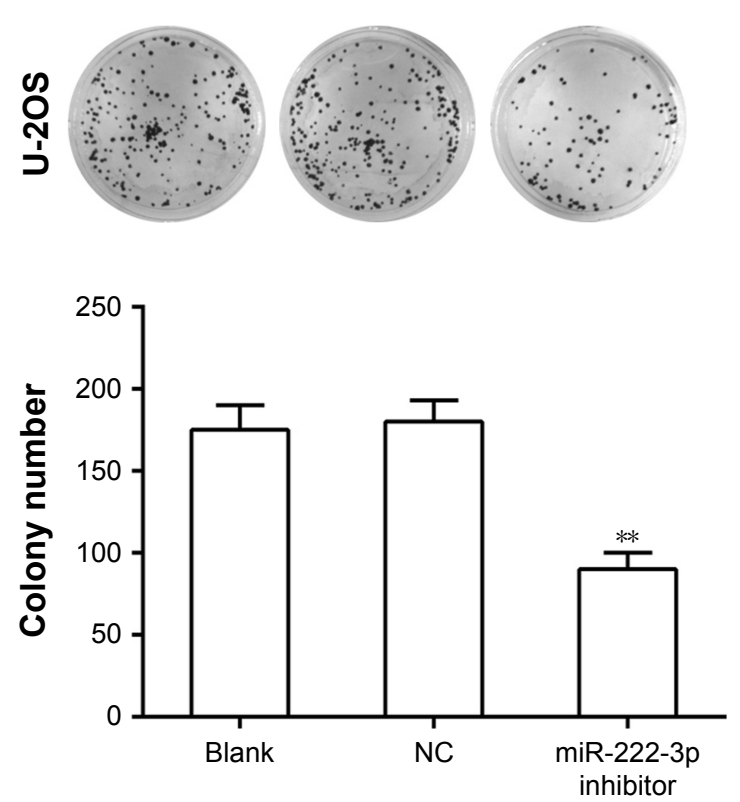

Figure 2 Inhibition of miR-222 in OS cells decreases their proliferation and colony formation.

Notes: (A, B) miR-222-3p inhibitor or NC was transfected into MG-63 and U-2OS cells, respectively, and miR-222-3p expression was measured using RT-qPCR. (C, D) Cell proliferation was assayed in MG-63 and U-2OS cells. Cells transfected with miR-222-3p inhibitor displayed decreased proliferation compared with the control. (E, F) Colony formation was assayed in MG-63 and U-2OS cells. ${ }^{* P}<0.05$; $* * P<0.01$.

Abbreviations: OS, osteosarcoma; RT-qPCR, reverse-transcription quantitative PCR; NC, control inhibitor. 

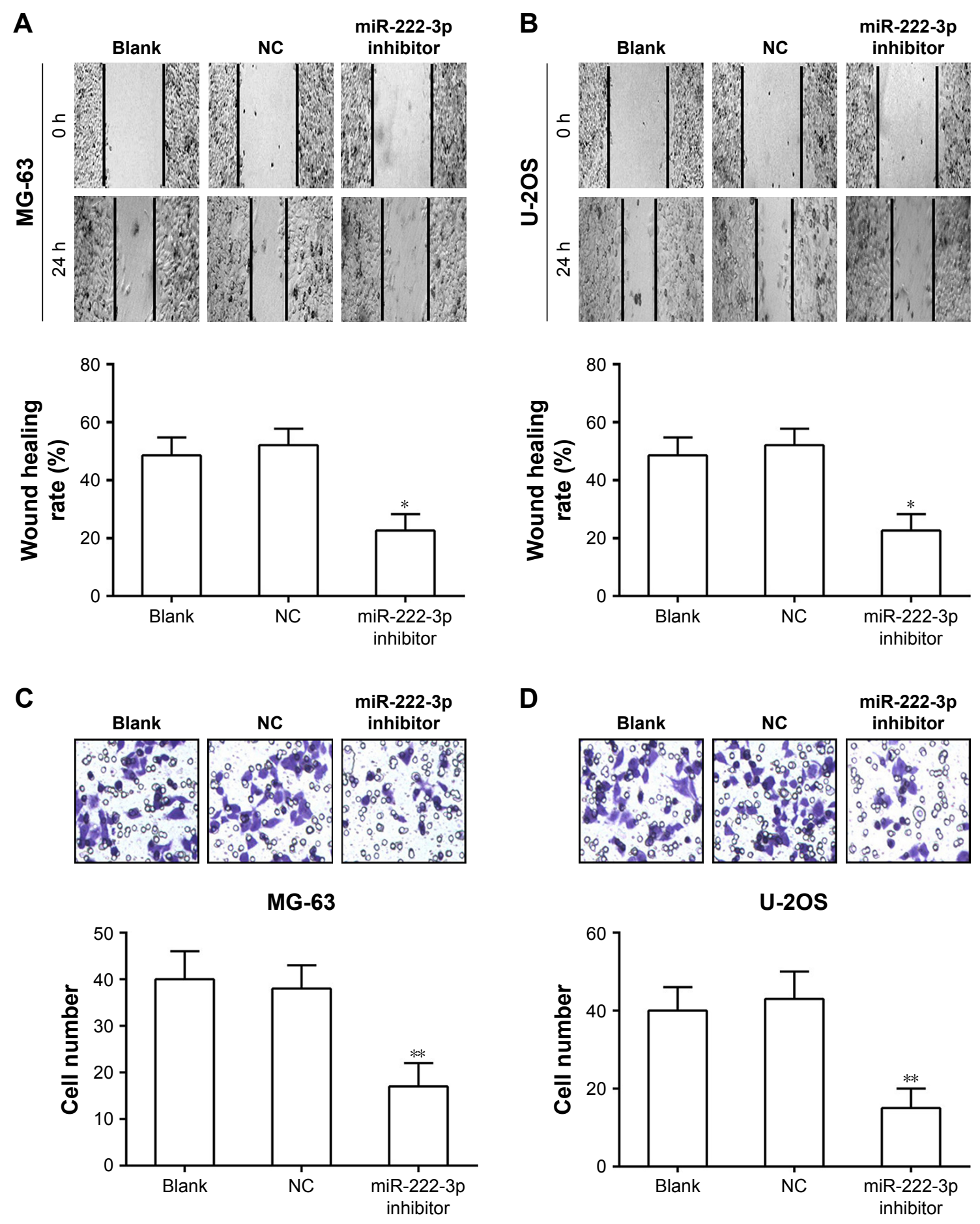

Figure 3 Inhibition of miR-222 suppresses migration and invasion in OS cells.

Notes: (A, B) Cell migration was determined by wound healing assay in MG-63 and U-2OS cells after transfection with miR-222-3p inhibitor or control inhibitor. (C, D) Transwell invasion assay was conducted in MG-63 and U-2OS cells. $* P<0.05$; $* * P<0.0$ I.

Abbreviation: OS, osteosarcoma.

normal tissues. Our data demonstrated that TIMP3 mRNA expression levels were markedly decreased in OS tissues compared with matched adjacent normal tissues (Figure 5A). Moreover, Spearman's correlation analysis revealed that TIMP3 mRNA expression was inversely correlated with miR-222-3p expression in OS tissues (Figure $5 \mathrm{~B} ; r=-0.754, P<0.01$ ).
TIMP3 is involved in miR-222-3p-

\section{mediated growth of OS cells}

To further explore the function of TIMP3 in miR-222-3pmediated proliferation of OS cells, the efficiency of TIMP3 siRNAs was first validated by Western blot analysis in the cells (Figure 6A). MTT and colony formation assays 


\section{A}

Position 2443-2449 of TIMP3 3'-UTR

hsa-miR-222-3p

TIMP3 3'-UTR mutant
5'...AAUUUAAAAUCAUUUAUGUAGCU...

| | | | |

3'...UGGGUCAUCGGUCUACAUCGA...

5'...AAUUUAAAAUCAUUUAACAUGCU...
B

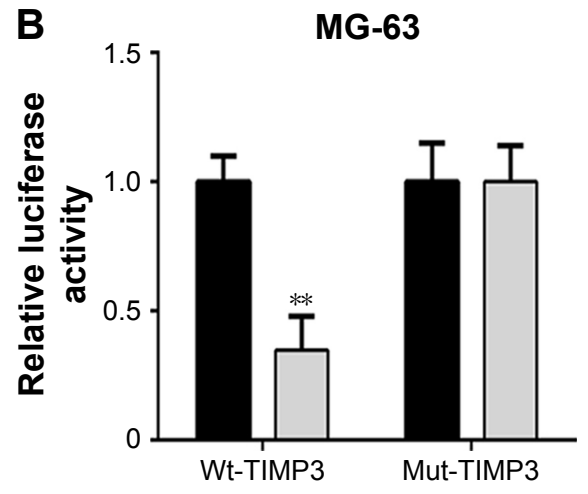

C U-20S

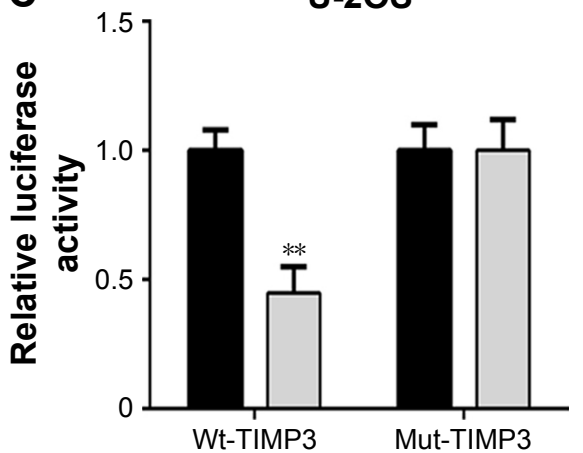

$\square$ NC $\square$ miR-222-3p

D

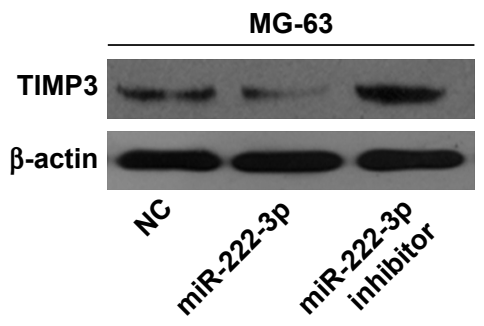

E

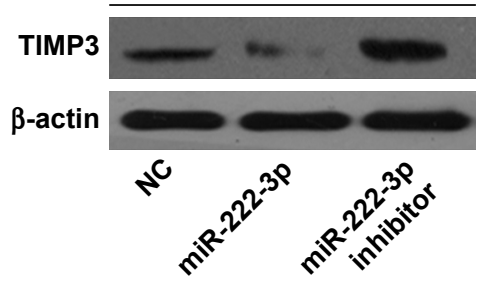

Figure 4 TIMP3 is a direct target of miR-222-3p.

Notes: (A) The suspected binding of mature human miR-222-3p with the wild-type 3'-UTR region of TIMP3 mRNA is shown. A mutated 3'-UTR of TIMP3 is also shown. (B, C) A dual-luciferase reporter assay was performed with MG-63 and U-2OS cells cotransfected with firefly luciferase constructs containing wild-type 3'-UTR region of TIMP3 (Wt-TIMP3) or mutated 3'-UTR region of TIMP3 (Mut-TIMP3) and miR-222-3p mimic or control. The relative luciferase activities were evaluated 24 hours after transfection $(* * P<0.05)$. (D, E) Osteosarcoma MG-63 and U-2OS cells were infected by miR-222-3p mimic, miR-222-3p inhibitor, or control. The protein expression levels of TIMP3 were evaluated by Western blotting.

Abbreviations: TIMP3, tissue inhibitor of metalloproteinases 3; UTR, untranslated region; NC, control inhibitor.

A

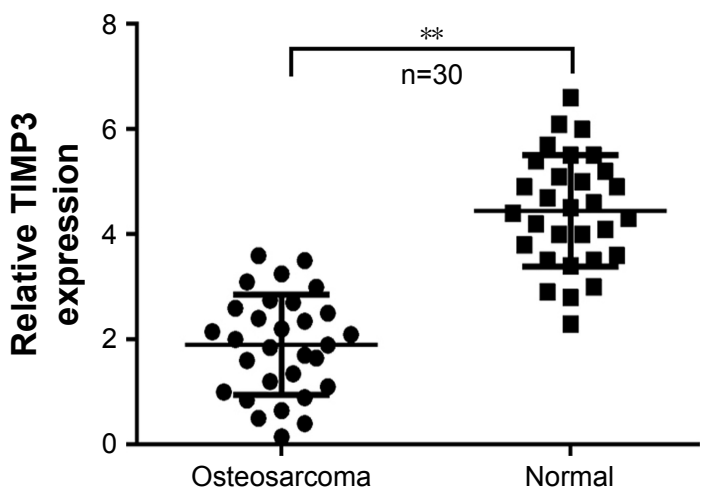

B

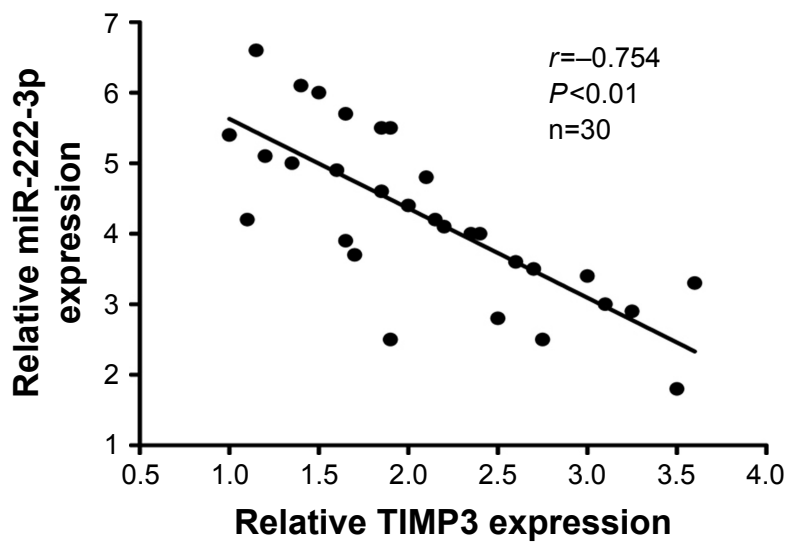

Figure 5 TIMP3 is inversely expressed with miR-222-3p in OS tissues.

Notes: (A) The gene expression levels of TIMP3 were compared between human clinical OS tissues and paired peritumoral tissues $(n=30$, $* * P<0.01)$. $(\mathbf{B})$ Correlation of miR-222-3p levels with TIMP3 mRNA levels was examined by RT-qPCR analysis in clinical OS tissues (Pearson's correlation coefficient, $r=-0.754$; $n=30 ; P<0.0$ ). Abbreviations: TIMP3, tissue inhibitor of metalloproteinases 3; OS, osteosarcoma; RT-qPCR, reverse-transcription quantitative PCR. 
A

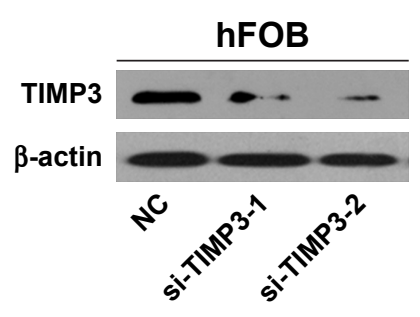

C

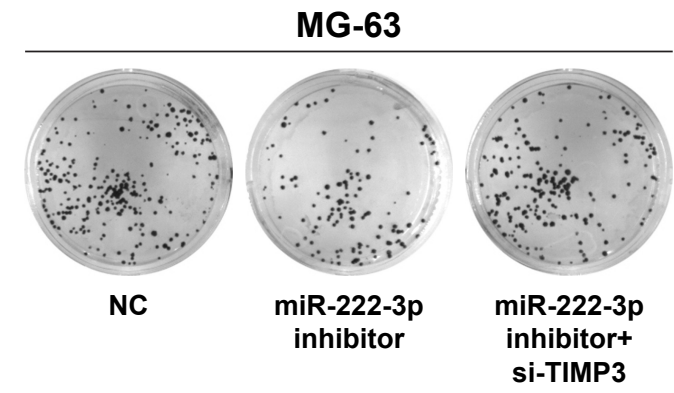

D

MG-63

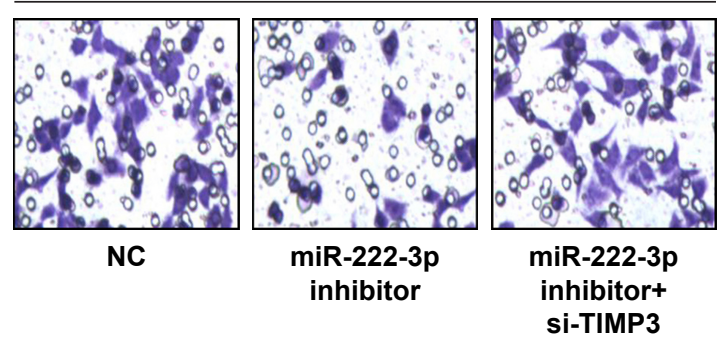

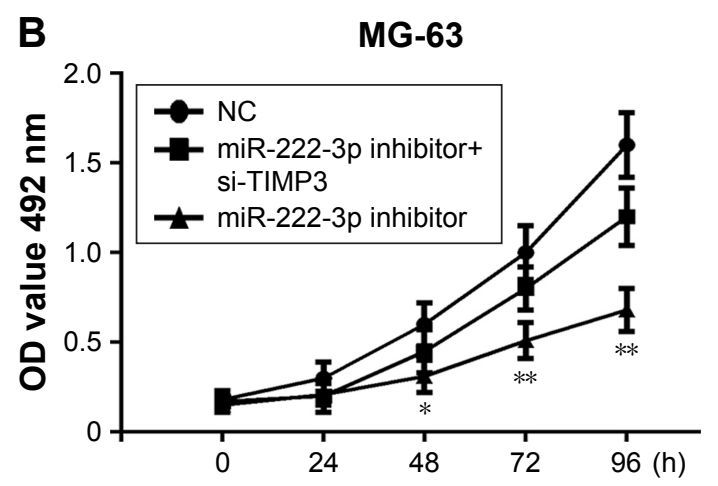
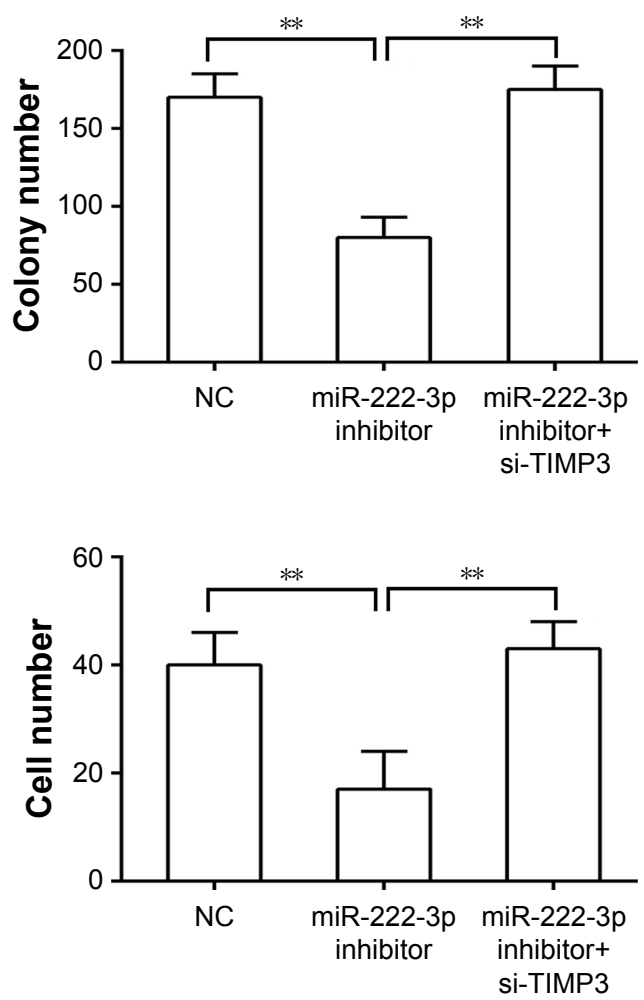

Figure 6 TIMP3 is involved in miR-222-3p-mediated regulation of OS cells.

Notes: (A) The interference efficiency of TIMP3 siRNAs (si-TIMP3-I or si-TIMP3-2) was examined by Western blotting in hFOB cells. (B) Effects of miR-222-3p inhibitor and miR-222-3p inhibitor+si-TIMP3 on MG-63 cell growth. (C) Effects of miR-222-3p inhibitor and miR-222-3p inhibitor+si-TIMP3 on MG-63 cell colony formation ability. (D) Effects of miR-222-3p inhibitor and miR-222-3p inhibitor+si-TIMP3 on MG-63 cell invasion ability. $* P<0.05$; $* * P<0.01$.

Abbreviations: TIMP3, tissue inhibitor of metalloproteinases 3; OS, osteosarcoma; NC, control inhibitor.

demonstrated that downregulation of TIMP3 could rescue the proliferation suppression of MG-63 cells by the miR-222-3p inhibitor (Figure 6B and C). In addition, the same effects were observed on the invasion capacity of MG-63 cells (Figure 6D). These results suggest that TIMP3 is involved in miR-222-3p-mediated regulation of OS cells.

\section{Inhibition of miR-222-3p reduced tumor growth in vivo}

Finally, we explored the potential involvement of miR222-3p in tumorigenesis though a MG-63 xenograft mouse model in vivo. Our data demonstrated that the tumors were smaller in the miR-222-3p inhibitor group compared with those in the miR-NC group (Figure 7A). Tumor size was found to be significantly smaller in the miR-222-3p inhibitor group compared with that in the miR-NC group (Figure 7B). Moreover, we examined miR-222-3p and TIMP3 expression levels in tumor tissue using RT-qPCR and Western blot analysis, respectively. Our data revealed that miR-222-3p expression was downregulated, whereas TIMP3 expression was obviously upregulated in the miR-222-3p inhibitor group compared with the miR-NC group (Figure 7C and D). 
A

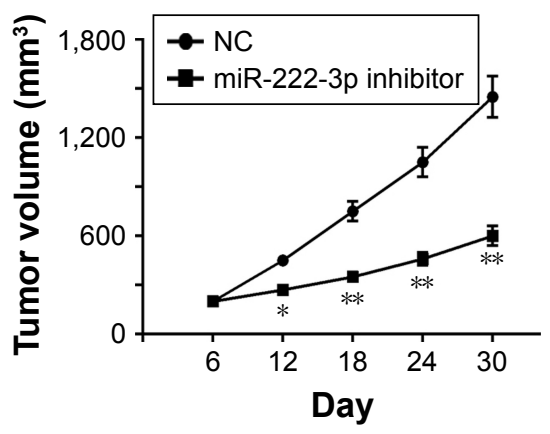

C

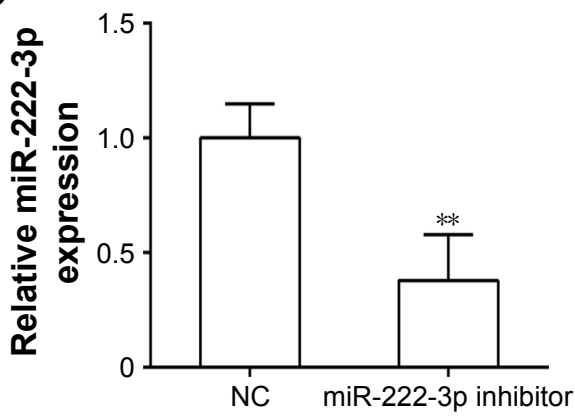

B

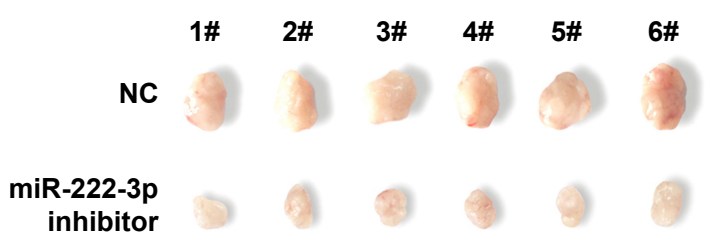

D

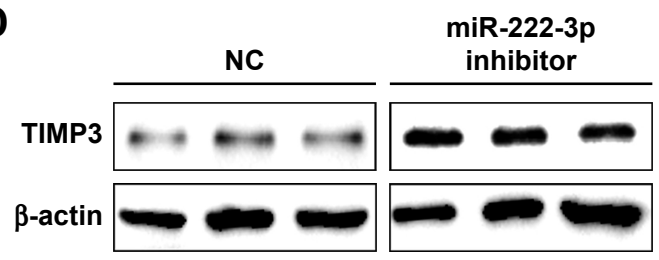

Figure 7 Inhibition of miR-222-3p reduces tumor growth in vivo.

Notes: (A) Tumor growth curves. (B) Photographs of tumor tissues. (C) miR-222-3p expression in tumor tissues was determined by RT-qPCR analysis. (D) TIMP3 protein expression in tumor tissues was determined by Western blotting. $\beta$-actin was used as an internal control. $* P<0.05 ; * * P<0.0$ I.

Abbreviations: RT-qPCR, reverse transcription-quantitative polymerase chain reaction; TIMP3, tissue inhibitor of metalloproteinases 3; NC, control inhibitor.

These findings indicate that miR-222-3p promotes tumor growth through suppressing TIMP3 expression in OS in vivo.

\section{Discussion}

OS is the most common primary malignant bone tumor, which most frequently occurs during childhood and adolescence, whereas the mechanism underlying its development remains elusive. ${ }^{20}$ Recent studies have demonstrated that ectopic expression of miRNAs has been observed in various cancers, including OS. ${ }^{10,11,21}$ Therefore, identification of specific miRNAs involved in the growth and metastasis of OS may provide new evidence pointing toward novel diagnostic and prognostic biomarkers and therapeutic targets for OS.

In the present study, we demonstrated that miR-222-3 $\mathrm{p}$ was upregulated in OS tissues and OS cell lines. In addition, inhibition of miR-222-3p can suppress OS cell proliferation, colony formation, and invasion in vitro. Taken together, these findings indicate that miR-222-3p may be a promising target for OS treatment. Various studies have demonstrated that the ectopic expression of miR-222-3p plays key roles in tumor growth and malignant behavior through modulating the expression of oncogenes and/or tumor suppressor genes. ${ }^{22-24}$ It has been reported that elevated expression of miR-222-3p in the serum may be a novel biomarker for screening OS patients and predicting poor prognosis. ${ }^{13}$ However, the detailed mechanism of miR-222-3p in OS tumorigenesis remains obscure and requires further investigation.

To determine the potential function of miR-222-3p in OS development, we analyzed the expression level of miR-222-3p in 30 OS paired tumor tissues using RT-qPCR. We found that miR-222-3p was markedly upregulated in OS tumor tissues and OS cell lines. Moreover, our data revealed that miR-222-3p increased OS cell proliferation by targeting TIMP3 in vitro. These results indicated that miR-222-3p may act as a tumor activator. It has been reported that miRNAs regulate various biological processes through modulating target genes. ${ }^{25,26}$ Therefore, we used three bioinformatics algorithms to predict possible target genes of miR-222-3p in OS cells. Our data demonstrated that a conserved miR-222-3p binding site was present in the 3 '-UTR of TIMP3. In addition, the luciferase assay confirmed that TIMP3 is a direct target gene of miR-222-3p in OS cells.

TIMP3, a member of the TIMP family of proteins, inhibits MMPs to limit degradation of the ECM. ${ }^{27}$ It has been reported that the expression level of TIMP3 was downregulated in various types of cancer. ${ }^{28,29}$ These studies indicated that TIMP3 may act as a potent tumor suppressor. Moreover, TIMP3 was verified as a target of several miRNAs, such 
as $\mathrm{miR}-21,{ }^{30} \mathrm{miR}-206,{ }^{31} \mathrm{miR}-181 \mathrm{~b},{ }^{32}$ and $\mathrm{miR}-373 .{ }^{33}$ In the present study, we observed that ectopic overexpression of miR-222-3p markedly suppressed TIMP3 expression levels. Furthermore, we investigated the TIMP3 expression level in OS tissues and it was found to be inversely correlated with miR-222-3p expression. In addition, downregulation of TIMP3 rescued the effect of miR-222-3p downregulation in OS cells. Taken together, our results indicate that miR-222-3p may act as a tumor activator, partially by targeting TIMP3 during the development of OS.

In conclusion, the present study identified miR-222-3p as a tumor growth activator in human OS through targeting TIMP3. These findings suggest that miR-222-3p and its target, TIMP3, may be a novel candidate target for OS therapeutics in the future.

\section{Acknowledgment}

This study was supported by the Scientific Research Project of Jilin Province (grant no JJKH20170052K).

\section{Disclosure}

The authors report no conflicts of interest in this work.

\section{References}

1. Anderson ME. Update on survival in osteosarcoma. Orthop Clin North Am. 2016;47(1):283-292.

2. Kager L, Zoubek A, Dominkus M, et al. Osteosarcoma in very young children: experience of the Cooperative Osteosarcoma Study Group. Cancer. 2010;116(22):5316-5324.

3. Lv YF, Dai H, Yan GN, Meng G, Zhang X, Guo QN. Downregulation of tumor suppressing STF cDNA 3 promotes epithelial-mesenchymal transition and tumor metastasis of osteosarcoma by the Wnt/GSK-3 $\beta / \beta$ catenin/Snail signaling pathway. Cancer Lett. 2016;373(2):164-173.

4. Ma B, Li M, Zhang L, et al. Upregulation of long non-coding RNA TUG1 correlates with poor prognosis and disease status in osteosarcoma. Tumour Biol. 2016;37(4):4445-4455.

5. Zhang Z, Zhang M, Chen Q, Zhang Q. Downregulation of microRNA-145 promotes epithelial-mesenchymal transition via regulating Snail in osteosarcoma. Cancer Gene Ther. 2017;24(2):83-88.

6. Bodles-Brakhop AM, Draghia-Akli R. DNA vaccination and gene therapy: optimization and delivery for cancer therapy. Expert Rev Vaccines. 2008;7(7):1085-1101.

7. Broadhead ML, Clark JC, Myers DE, Dass CR, Choong PF. The molecular pathogenesis of osteosarcoma: a review. Sarcoma. 2011;2011:1-12.

8. Wang S, Balasundaram G. Potential cancer gene therapy by baculoviral transduction. Curr Gene Ther. 2010;10(3):214-225.

9. Filipowicz W, Bhattacharyya SN, Sonenberg N. Mechanisms of posttranscriptional regulation by microRNAs: are the answers in sight? Nat Rev Genet. 2008;9(2):102-114.

10. Zhou H, Zhang M, Yuan H, Zheng W, Meng C, Zhao D. MicroRNA-154 functions as a tumor suppressor in osteosarcoma by targeting Wnt5a. Oncol Rep. 2016;35(3):1851-1858.

11. Pu Y, Zhao F, Li Y, et al. The miR-34a-5p promotes the multichemoresistance of osteosarcoma via repression of the AGTR1 gene. BMC Cancer. 2017;17(1):1-9.

12. Feng G, Shi H, Li J, et al. MiR-30e suppresses proliferation of hepatoma cells via targeting prolyl 4-hydroxylase subunit alpha-1 (P4HA1) mRNA. Biochem Biophys Res Commun. 2016;472(3):516-522.
13. Zhang Q, Wang S, Wang Y, du Y, Fu X. Serum level of microRNA222 acts as a diagnostic and prognostic biomarker for osteosarcoma patients. Int J Clin Exp Pathol. 2016;9(4):4843-4848.

14. Han XG, Li Y, Mo HM, et al. TIMP3 regulates osteosarcoma cell migration, invasion, and chemotherapeutic resistances. Tumour Biol. 2016;37(7):8857-8867.

15. Arpino V, Brock M, Gill SE. The role of TIMPs in regulation of extracellular matrix proteolysis. Matrix Biol. 2015;44-46(1):247-254.

16. Lin $\mathrm{H}$, Zhang $\mathrm{Y}$, Wang $\mathrm{H}$, et al. Tissue inhibitor of metalloproteinases-3 transfer suppresses malignant behaviors of colorectal cancer cells. Cancer Gene Ther. 2012;19(12):845-851.

17. Adissu HA, Mckerlie C, di Grappa M, et al. Timp3 loss accelerates tumour invasion and increases prostate inflammation in a mouse model of prostate cancer. Prostate. 2015;75(16):1831-1843.

18. Kong L, Zhang P, Li W, et al. KDM1A promotes tumor cell invasion by silencing TIMP3 in non-small cell lung cancer cells. Oncotarget. 2016;7(19):27959-27974.

19. van der Velden PA, Zuidervaart W, Hurks MH, et al. Expression profiling reveals that methylation of TIMP3 is involved in uveal melanoma development. Int J Cancer. 2003;106(4):472-479.

20. Geller DS, Gorlick R. Osteosarcoma: a review of diagnosis, management, and treatment strategies. Clin Adv Hematol Oncol. 2010;8(10):705-718.

21. Zhang R, Yan S, Wang J, et al. MiR-30a regulates the proliferation, migration, and invasion of human osteosarcoma by targeting Runx2. Tumour Biol. 2016;37(3):3479-3488.

22. Yang CJ, Shen WG, Liu CJ, et al. miR-221 and miR-222 expression increased the growth and tumorigenesis of oral carcinoma cells. J Oral Pathol Med. 2011;40(7):560-566.

23. Quintavalle C, Garofalo M, Zanca C, et al. miR-221/222 overexpession in human glioblastoma increases invasiveness by targeting the protein phosphate РТP $\mu$. Oncogene. 2012;31(7):858-868.

24. Li Q, Shen K, Zhao Y, et al. MicroRNA-222 promotes tumorigenesis via targeting DKK2 and activating the Wnt/ $\beta$-catenin signaling pathway. FEBS Lett. 2013;587(12):1742-1748.

25. Zhang W, Lu Z, Gao Y, Ye L, Song T, Zhang X. MiR-520b suppresses proliferation of hepatoma cells through targeting ten-eleven translocation 1 (TET1) mRNA. Biochem Biophys Res Commun. 2015;460(3): 793-798.

26. Zheng L, Zhang Y, Liu Y, et al. MiR-106b induces cell radioresistance via the PTEN/PI3K/AKT pathways and p21 in colorectal cancer. J Transl Med. 2015;13(1):252-265.

27. Wang CY, Liou JP, Tsai AC, et al. A novel action mechanism for MPT0G013, a derivative of arylsulfonamide, inhibits tumor angiogenesis through up-regulation of TIMP3 expression. Oncotarget. 2014; 5(20):9838-9850.

28. Fan B, Jin X, Shi Y, et al. Expression and significance of TIMP-3, PACAP and VIP in vaginal wall tissues of patients with stress urinary incontinence. Exp Ther Med. 2017;13(2):624-628.

29. Diao Y, Fu H, Wang Q. MiR-221 exacerbate cell proliferation and invasion by targeting TIMP3 in papillary thyroid carcinoma. Am J Ther. 2017;24(3):e317-e328.

30. Nagao Y, Hisaoka M, Matsuyama A, et al. Association of microRNA-21 expression with its targets, PDCD4 and TIMP3, in pancreatic ductal adenocarcinoma. Mod Pathol. 2012;25(1):112-121.

31. Fu X, Zeng L, Liu Z, Ke X, Lei L, Li G. MicroRNA-206 regulates the secretion of inflammatory cytokines and MMP9 expression by targeting TIMP3 in Mycobacterium tuberculosis-infected THP-1 human macrophages. Biochem Biophys Res Commun. 2016;477(2):167-173.

32. Heath JM, Fernandez Esmerats J, Khambouneheuang L, Kumar S, Simmons R, Jo H. Mechanosensitive microRNA-181b regulates aortic valve endothelial matrix degradation by targeting TIMP3. Cardiovasc Eng Technol. 2018;9(2):141-150.

33. Liu W, Li M, Chen X, et al. MicroRNA-373 promotes migration and invasion in human esophageal squamous cell carcinoma by inhibiting TIMP3 expression. Am J Cancer Res. 2016;6(1):1-14. 
OncoTargets and Therapy

\section{Publish your work in this journal}

OncoTargets and Therapy is an international, peer-reviewed, open access journal focusing on the pathological basis of all cancers, potential targets for therapy and treatment protocols employed to improve the management of cancer patients. The journal also focuses on the impact of management programs and new therapeutic agents and protocols on

perspectives such as quality of life, adherence and satisfaction. The manuscript management system is completely online and includes a very quick and fair peer-review system, which is all easy to use. Visit http://www.dovepress.com/testimonials.php to read real quotes from published authors.

Submit your manuscript here: http://www.dovepress.com/oncotargets-and-therapy-journal 